\title{
Care stress in caregivers of disabled stroke patients: a cross-sectional survey
}

\author{
Xiaotan Wu ${ }^{1,2 \#}$, Yu Liang ${ }^{1 \#}$, Bijin Zheng ${ }^{3 \#}$, Hui Wang ${ }^{1}$, Min Ning ${ }^{1}$, Haihua Zheng ${ }^{1}$, Baoxin Shi ${ }^{4}$ \\ ${ }^{1}$ Cadre sanatorium of Hainan \& Geriatric hospital of Hainan, Haiou, China; ${ }^{2}$ School of Nursing, Tianjin Medical University, Tianjin, China; ${ }^{3}$ Second \\ Hospital of Tianjin Medical University, Tianjin, China; ${ }^{4}$ Hospice Research Center, Tianjin Medical University, Tianjin, China \\ Contributions: (I) Conception and design: X Wu, B Zheng, B Shi; (II) Administrative support: Y Liang, H Wang, M Ning, H Zheng; (III) Provision of \\ study materials or patients: Y Liang, H Wang, M Ning; (IV) Collection and assembly of data: X Wu, Y Liang; (V) Data analysis and interpretation: X \\ Wu, B Shi; (VI) Manuscript writing: All authors; (VII) Final approval of manuscript: All authors. \\ "The authors contributed equally to this work. \\ Correspondence to: Baoxin Shi. Hospice Research Center, Tianjin Medical University, Tianjin 300070, China. Email: shibaoxin88@126.com.
}

Background: A cross-sectional survey was performed on the family members of disabled stroke survivors, those who are both the patient's medical authorizer and caregiver, to identify the sources of the caring stress and inform appropriate interventions.

Methods: A total of 242 family members of stroke patients, who were treated in a tertiary geriatric hospital in Haikou, the capital city of Hainan Province, were enrolled in the current study by using convenience sampling. Questionnaire forms were used to investigate care stress caused by family members' current care activities and analyze its causes.

Results: The care stress of the family members was moderately positively correlated with financial pressure $(\mathrm{r}=0.476, \mathrm{P}<0.01)$. Family members' educational background, financial pressure, the times of hospitalizations, the dependency of stroke patients, and the degree of family members' participation in treatment decisionmaking during the patient's hospital stay were the primary sources of the caring stress $(\mathrm{P}<0.05$ or $\mathrm{P}<0.01)$.

Conclusions: A substantial proportion of family members are under high stress when caring for disabled stroke patients. Strategies including knowledge training, empowerment, home-based rehabilitation, and information and emotional support may effectively relieve the caring stress, to improve the family support of patients, improve the quality of life of both patients and caregivers, and enhance the rehabilitation effectiveness.

Keywords: Stroke; family members; stress; self-care; family support; disability

Submitted Jun 17, 2020.Accepted for publication Jul 14, 2020.

doi: 10.21037/apm-20-1369

View this article at: http://dx.doi.org/10.21037/apm-20-1369

\section{Introduction}

Disability affects $75 \%$ of stroke survivors. Long-term care and support from family caregivers are needed due to the long disease course and slow recovery (1). However, long-term care of stroke patients can quickly increase the burden of family caregivers, leading to increased negative emotions and decreased quality of life $(2,3)$. In turn, the family members' stress will directly affect their attitudes towards the patient and the quality of care (4). Poststroke depression, poststroke anxiety and post-traumatic stress disorder, which are common among stroke survivors and take a heavy toll on stroke survivors. The clinical and research of stroke rehabilitation mainly focuses on motor disorders, speech and language deficits, and cognitive dysfunction, while psychological stress is largely ignored. The high-stress levels of the patient's family members not only reduce their quality of life but also directly affects the patient's emotions, which affects the patient's compliance with rehabilitation and treatment and even causes or aggravates the patient's depression (5).

Previous studies $(2,3)$ mostly focused on care-stress in stroke care-givers, while few studies focused on the stress caused by the 
patient's family members in the process of caring for the patient, In the current study, we surveyed the stress and its sources in the family members (and the caregivers) of disabled stroke patients, with an attempt to further improve the family support and the rehabilitation effect of disabled stroke patients.

We present the following article in accordance with the MDAR reporting checklist (available at http://dx.doi. org/10.21037/apm-20-1369).

\section{Methods}

\section{Subjects}

Family members of disabled stroke patients in a tertiary geriatric hospital in Haikou, the capital city of Hainan Province, from November 2018 to January 2019, were enrolled in this survey by using convenience sampling. The inclusion criteria included: (I) aged $\geq 18$ years; (II) signer of the informed consent forms during patient's hospitalization and primary caregiver during the hospital stay and home-based care; (III) with clear consciousness as well as complete cognitive and behavioral abilities, and being able to comprehend the text of the questionnaire; (IV) the patients he/she cared for were stroke patients who were dependent [with a Barthel Index (BI) of Activities of Daily Living (ADL) score of $<100$ points]; and (V) the caregiver was in excellent mental state and were willing to cooperate with the questionnaire survey by signing an informed consent form. The exclusion criteria included: (I) with poor mental state, unable to communicate effectively, and unable to comprehend the text of the questionnaire; (II) the patient he/she cared for were dependent (on the BI); after the informed consent was obtained from the heads of each neurology department, the eligible family members are invited to join the study. The investigators explained the study background and objectives of the subjects, and informed consent was obtained from all the respondents. This study was approved by the medical Ethics Committee of Hainan Cadres Sanatorium (Hainan Geriatric Hospital). All procedures performed in this study involving human participants were in accordance with the Declaration of Helsinki (as revised in 2013).

\section{Research tools}

\section{General information forms}

The self-designed questionnaire on the general information included two forms: general information of the family members and general information of the stroke patient.

The general information form for the family members included age, gender, current residence, education background, religious beliefs, marital status, occupation, relationship with the patient, the experience of hospitalization, degree of participation in the patient's in-hospital care, the proportion of payment for patient's hospitalization fees, and degree of participation in the patient's treatment decision-making during the hospital stay.

The contents of the patient's general information form included age, educational background, number of hospitalizations, the proportion of the medical costs covered by the medical insurance system, and the duration of the disability.

\section{BI}

Developed by Mshoney and Barthel in 1965, the BI is a measure of independence in ADL for patients in American rehabilitation centers (6). It is also the most used scale for assessing the ADL of patients in China and has shown excellent reliability and efficiency in stroke patients (7). BI consists of 10 items assessing the ability to achieve activities without assistance. It evaluates the ability of feeding, moving from wheelchair to bed and returning, doing personal toilet, getting on and off the toilet, bathing self, walking on a level surface, ascending and descending stairs, dressing, controlling bowels, and controlling the bladder. Scoring ranges from 0 (completely dependent) to 100 (completely independent) with intervals of 5 points. The BI scores are classified into 4 groups: 0-40 is a complete dependency, 45-60 is a moderate dependency, 65-95 is a mild dependency, and 100 is no dependency. Two trained professionals used $\mathrm{BI}$ to evaluate the ADL of the patients while conducting a questionnaire-based survey on the family members. The patients' self-care abilities were graded according to the BI scores.

\section{Family member's self-perceived stress evaluation form}

This form was used to assess the stress arising from patient care and cost payment. The respondents were asked to select a stress option on the form according to their conditions.

(I) Care stress: according to the stress experienced during patient care. There were four options: "no stress," "stress does exist but has not affected my work and life" "the heavy stress has affected my work and life," and "the stress is extremely heavy, and my work and life have been seriously affected, and 
sometimes I cannot work or live in a normal way."

(II) Financial pressure: according to the stress experienced by the family members when paying medical costs, there were four options: "There is no financial pressure," "I feel the financial pressure somehow," "The financial pressure is quite large but is still manageable," and "We are under extremely heavy pressure, and we are unable to pay the medical bills."

\section{Data collection}

After obtaining the approval from the hospital ethics committee of our center and with the support of the department heads, we identified the subjects and invited them to fill the questionnaire forms when the patients were receiving infusion treatment. Two uniformly trained investigators collected the data. They explained the questionnaire content and survey purpose to the family members to obtain their cooperation. The family members were told that all the questions were not supposed to have right or wrong answers, and the respondents were anonymous. They were also assured that the filling of the questionnaire would not affect the management of the patient. A total of 260 questionnaire forms were distributed after obtaining informed consent from the family members. Eighteen family members withdrew from the survey for other reasons, and finally, 242 valid forms (93.08\%) were recovered.

\section{Statistical analysis}

Data were double-entered by two different researchers into Microsoft Office Excel 2007 spreadsheets and then analyzed by using the SPSS 19.0 software package. Count data are expressed by frequencies and proportions and measurement data by $\bar{X} \pm s$. According to the general information of patients and their family members, Spearman correlation analysis was conducted on the caring stress and financial pressure of the caregivers to identify the sources of care stress. According to the general information, patient's ADL, caregiver's participation in treatment decision-making, univariate analysis was performed on the degree of care stress perceived by the caregiver to find out the possible causes of the stress. Then a multivariate Logistic regression analysis was performed on the possible sources to identify the sources of care stress and their impacts finally.

\section{Results}

\section{The general information of caregivers and patients}

A total of 242 pairs of patients and their immediate relatives (i.e., the caregivers) were surveyed in this study. The general information of the caregivers was as follows: aged $(47.89 \pm 13.139)$ years; 128 males $(52.9 \%)$ and 114 females $(47.1 \%) ; 80(33.1 \%)$ resided in cities, 82 $(33.9 \%)$ in towns, $80(33.1 \%)$ in rural areas; the education background included primary school or lower in 31 cases $(12.8 \%)$, middle school in 77 cases $(31.8 \%)$, high school/ polytechnic school in 77 cases (31.8\%), college or above in 57 cases $(23.5 \%) ; 220$ cases $(90.9 \%)$ had religious beliefs and 22 cases $(9.1 \%)$ had no religious beliefs; the marital status included unmarried in 11 cases (4.5\%), married with children in 223 cases $(92.1 \%)$, married without children in 6 cases $(2.5 \%)$, and divorced in 2 cases $(0.8 \%) ; 39$ cases $(16.1 \%)$ were medical staff, media workers, or education workers and 203 persons (83.9\%) had other occupations; their relationships with the patients were spouses in 60 persons $(24.8 \%)$, children in 170 persons $(70.2 \%)$, parents in 4 persons (1.7\%), and siblings in 8 cases (3.3\%); 104 respondents (43.0\%) had hospitalization experience; during the patients' hospitalization, 72 respondents $(29.8 \%)$ cared for the patients alone for 24 hours, and 161 (66.5\%) had rotating family members to co-care the patients; during the home-based care, 67 family members $(27.7 \%)$ were the primary caregivers for the patients and $169(69.8 \%)$ offered care with other family members; 81 respondents (33.5\%) paid for the hospitalization costs of patients independently, 149 respondents (61.6\%) shared the payment with other family members, and 12 respondents (5.0\%) did not need to worry about the payment.

The general information of the patients was as follows: aged $(69.60 \pm 10.812)$ years old; the education background was primary school or lower in 94 cases $(38.8 \%)$, middle school in 67 cases $(27.7 \%)$, and high school or higher in 81 cases $(33.5 \%)$; the proportion of reimbursable hospitalization expenditure was $100 \%$ in 3 cases $(1.2 \%)$, $80-99 \%$ higher in 132 cases $(54.5 \%), 60-79 \%$ in 64 cases (26.4\%), $1-60 \%$ in 37 cases $(15.3 \%)$, and $0 \%$ in 6 cases (2.5\%). Also, 56 patients (23.1\%) were hospitalized due to stroke for the first time, 60 patients $(24.8 \%)$ were readmitted for the first time, and 126 patients $(52.1 \%)$ had been readmitted for 2 or more times; the dependency had lasted for less than 3 months in 121 patients (50.0\%), $3-12$ months in 74 patients (30.5\%), and more than 1 year in 47 patients (19.4\%); according to BI, 160 patients $(66.1 \%)$ were mildly dependent, 40 (16.5\%) were and moderately 
dependent, and $42(17.4 \%)$ were severely dependent. The ADL scores were $(86.66 \pm 9.611)$ in mildly dependent patients, $(53.00 \pm 6.284)$ in moderately dependent patients, and $(23.10 \pm 11.367)$ in severely dependent patients.

\section{Stress of family members during the caring of disabled stroke patients}

For family members who directly cared for disabled stroke patients, $22.7 \%$ of the respondents felt that the caring stress

Table 1 Care stress and financial pressure from patients' hospitalization perceived by the family members $(n=242)$

\begin{tabular}{lc}
\hline Item & $\mathrm{n}(\%)$ \\
\hline Care stress & $53(21.9)$ \\
No & $56(23.1)$ \\
A little & $78(32.2)$ \\
High & $55(22.7)$ \\
Very high & $33(13.6)$ \\
Financial pressure from patients' hospitalization \\
No & $54(22.3)$ \\
A little & $90(37.2)$ \\
High & $65(26.9)$ \\
Very high & \\
\hline
\end{tabular}

Table 2 Sources of care stress among caregivers $(n=242)$

\begin{tabular}{lcc}
\hline Item & Care stress & P value \\
\hline Family member's age & 0.180 & 0.005 \\
Financial pressure & 0.476 & 0.000 \\
Patient's dependency & 0.258 & 0.000 \\
\hline
\end{tabular}

was very high, and $26.9 \%$ of the respondents replied the financial pressure from hospital stay was burdensome. The proportion of family members who had financial pressure was more extensive than those with care stress (Table 1).

\section{Sources of care pressure and financial pressure}

Spearman correlation analysis showed that the caring stress was positively correlated with the family member's age, the financial pressure from disease treatment, and the patient's dependency (Table 2).

Spearman correlation analysis showed that financial pressure from hospital expenditure was positively correlated with the proportion of out-of-pocket expenditures, stroke patients' dependency, and family member's care stress but was negatively correlated with the proportion of hospitalization reimbursement and the family member's education background (Table 3).

\section{Univariate analysis and multivariate Logic regression analysis of the sources of care stress}

Univariate analysis was performed with the general information and the financial pressure as independent variables and the degree of care stress as a dependent variable. The Pearson chi-square test showed that the age of family members, family members' relation with patient, financial pressure, the participation of family members in in-hospital care, and patient's dependency affected the caring stress (Table 4).

A multivariate Logic regression model was set up with the indicators screened by the univariate analysis as independent variables and the caring stress as the dependent variable. The likelihood ratio test statistics were $\chi^{2}=268.258$ and $\mathrm{P}<0.01$, suggesting that the model fit the data well.

Compared with family members with an education degree of "College or higher," those with an education

Table 3 Sources of financial pressure among caregivers $(n=242)$

\begin{tabular}{lcc}
\hline Item & Financial pressure & P value \\
\hline Proportion of out-of-pocket expenditures & 0.229 & 0.000 \\
Proportion of hospitalization reimbursement & -0.157 & 0.014 \\
Family member's education background & -0.203 & 0.001 \\
Care stress & 0.476 & 0.000 \\
Patient's dependency & 0.133 & 0.039 \\
\hline
\end{tabular}


Table 4 Univariate analysis of the sources of care stress

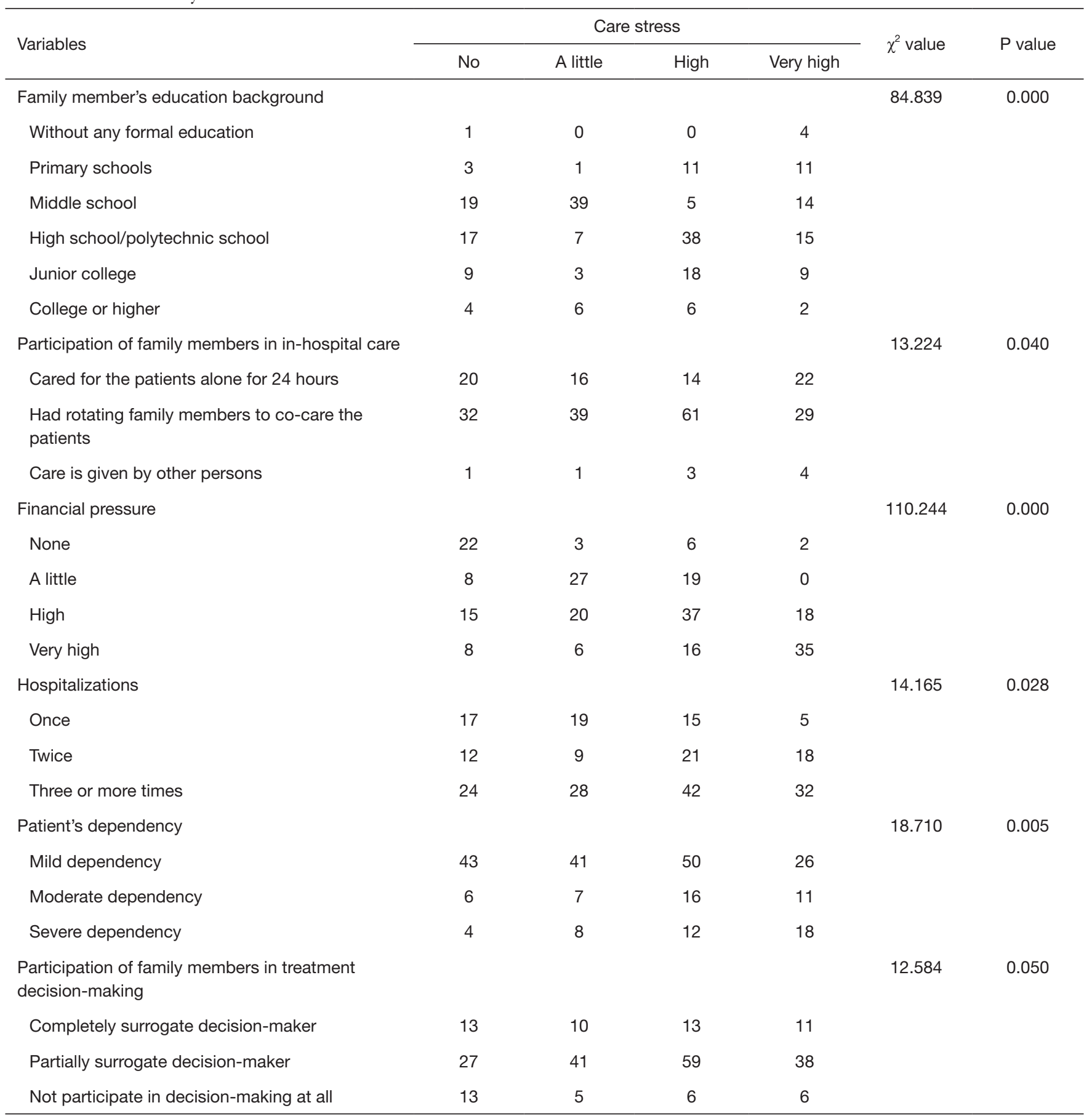

Note: only the statistically significant items are listed.

degree of "Without any formal education" or "Primary school" had 62.273 times and 25.632 times possibility of developing high stress, respectively. Family members who had no financial pressure arising from hospitalization expenditures had only 0.006 times the possibility of becoming stressed than those who had "Very high" financial pressure. Fewer hospitalizations were associated with lower care stress. The ratio of family members who responded 
Table 5 Multivariate logistic regression analysis of sources of care stress

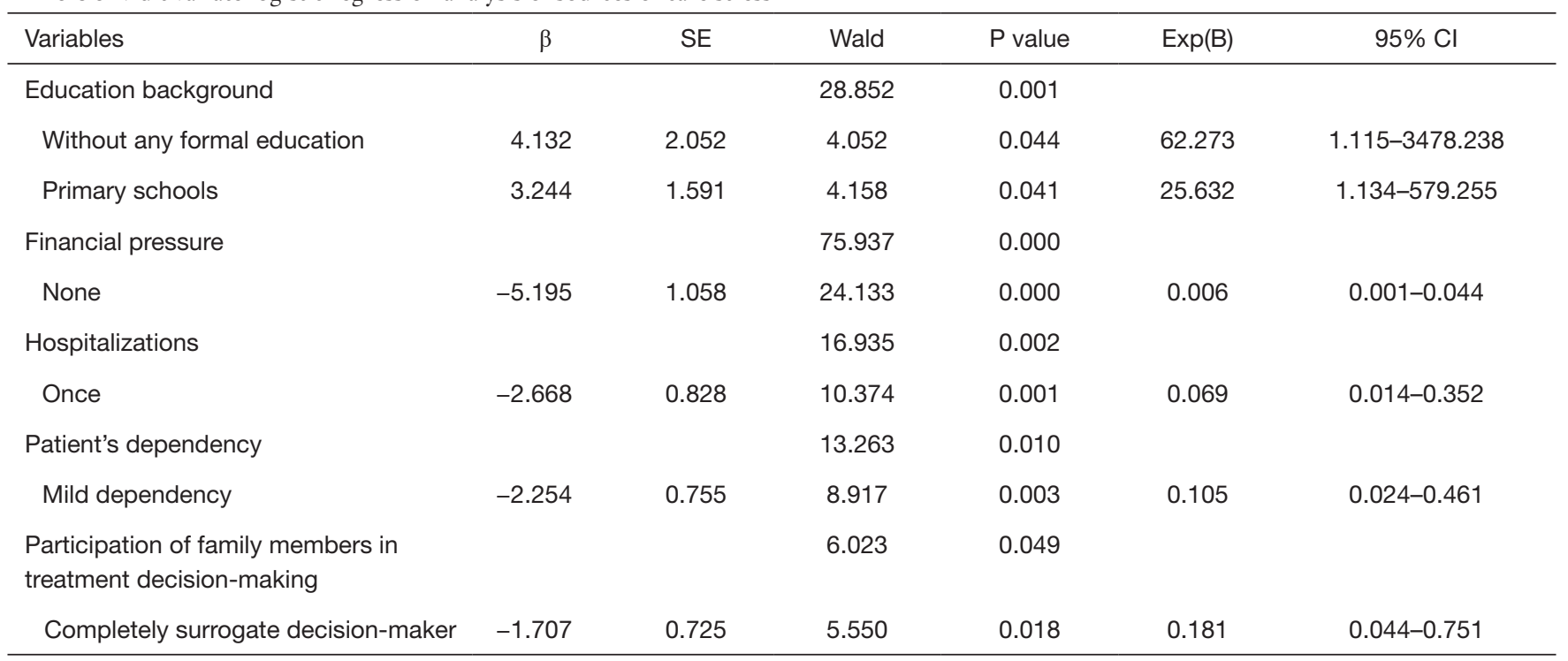

that there was "No" stress was 0.069:1 between family members of patients who were admitted for the first time and those of patients who had been admitted for three or more times. Lower patient's dependency was associated with lower care stress. The family members of patients with mild dependency had 0.105 times the possibility of perceiving care stress than the family members of patients with severe dependency. During the patient's hospitalization, the family members who served as the surrogate decision-makers had 0.181 times possibly of feeling care stress than the family members of patients who made treatment decisions independently (Table 5).

\section{Discussion}

Family members have varying degrees of stress during the hospitalization of a stroke patient

In China, close family ties are much more common. Caring for a sick family member is the responsibility of all the other members, and supports from the family is vital for the rehabilitation of the patient, both mentally and physically. However, family caregivers often cannot meet the needs of the patient due to insufficient professional training and knowledge and insufficient support from social resources. These caregivers are often under tremendous stress, and, in turn, the family members' stress will directly affect their attitudes towards the patient and the quality of care (4). Research has shown that stroke caregivers and patients have a high degree of dependence. Caregivers with low stress and negative emotions, good physical and mental health, and high quality of life can reduce patients' perception of strokerelated symptoms/signs, promote their mental health, and improve their quality of life (8). In our current survey, most stroke caregivers $(78.1 \%)$ complained of care stress, and 55 $(22.7 \%)$ of them felt the caring stress was very high, which was consistent with the finding $(79.2 \%)$ of Yang et al. (9) but slightly higher than that $(62.5 \%)$ of Wang et al. (10). Such differences may be explained by the geographical difference of the research populations or by the biases caused by the different educational backgrounds, care ability, and socioeconomic status. Although long-term care for patients brings heavy burdens, stress, and other negative feelings to the caregivers, the caregivers may have positive experiences, including satisfaction, improved self-esteem, and gratitude during the care (11). Therefore, in addition to the negative feelings of caregivers and the corresponding interventions, the positive feelings of caregivers should also be further investigated to understand the mechanisms and sources of such positive feelings; accordingly, measures can be taken to help the caregivers strengthen their positive feelings and alleviate the caring stress $(12,13)$. Maintaining care routines, keeping a sense of humor, and accumulating a wealth of care experience can cause caregivers more likely to experience positive experiences during the care of stroke patients and, to a certain extent, ease the impacts of the caring stress and negative feelings on the quality of life of the caregivers. 


\section{Multiple sources of care stress}

In a previous study (5), the caregiver's age and education level, daily care time, the financial burden caused by the disease, medical insurance payment mode, the severity of the patient's disease, and the caregiver's knowledge of stroke affected the care ability. Weak care ability increases care stress. In our current study, the caregivers of stroke patients were their children $(70.5 \%)$, followed by their spouses $(24.1 \%)$. Up to $45.4 \%$ of the caregivers were older than 50 years, and those aged $\geq 65$ years accounted for $22 \%$. Two caregivers were over 80 years. Spearman correlation analysis showed that care stress increased with age $(\mathrm{r}=0.180$, $\mathrm{P}<0.01)$. Understandably, the old caregivers have decreased physical functions and care abilities; so, their care stress increases.

Compared with family members with an education degree of "College or higher," those with an education degree of "Without any formal education" or "Primary school" had significantly higher times the possibility of developing high stress (both $\mathrm{P}<0.01$ ). These results may be because caregivers with low educational backgrounds have low awareness of the disease and weak ability to learn and comprehend new knowledge, and poor capability in access to and utilize medical information resources (5). Medical staff should repeatedly educate these caregivers with simple and easy-to-understand language to improve their care abilities. Empowerment programs and collaborative nursing interventions can significantly improve the care ability of the caregivers of stroke patients and effectively improve the quality of life and ease the caring stress in these caregivers. Correlation analysis also showed the family members' educational background was negatively correlated with the financial pressure $(r=-0.203, \mathrm{P}<0.01)$. Family members with a low education degree were more likely to experience high financial pressure when caring for stroke patients, which may be due to the poor knowledge and low ability of these family members in accessing financial resources. Also, financial pressure was positively correlated with care stress $(\mathrm{r}=0.476, \mathrm{P}<0.01)$, so how to lower the financial pressure is very important. The government authorities should pay special attention to stroke patients and their caregivers by optimizing relevant welfare policies, easing the stress status of caregivers, and improving the quality of care. Also, rehabilitation hospitals, governments, volunteers, and other stakeholders may organize personnel to offer home-based rehabilitation for discharged patients, improve the ability of patients' family members to care for patients at home for economic reasons.

Logic regression analysis showed that the increase in the number of hospitalizations of stroke patients increased the caring stress $(\mathrm{P}<0.01)$, and family members who had no financial pressure when paying for the patient's disease were less likely to have a high-stress status when caring for patients. Spearman correlation analysis also found that the caring stress was moderately positively correlated with the financial pressure arising from the payment for the disease $(\mathrm{r}=0.476, \mathrm{P}<0.01)$. A high recurrence rate characterizes stroke and frequent hospitalizations; its long disease course and slow recovery require intensive rehabilitation services and high treatment costs (14). Research has shown that $10 \%$ of stroke patients will be readmitted within 30 days of discharge (15). Repeated hospitalizations bring a considerable financial burden to the patient's family, along with the caring stress (16). In our current study, 225 patients $(94.8 \%)$ had their hospitalization expenditures paid by their family members. The financial pressure for paying the hospital expenditures was "High" for $64.1 \%$ of family members and "Very high" for $26.9 \%$ of the respondents. The financial pressure was positively correlated with the proportion of family member's payment for patient's hospitalization fees and negatively correlated with the proportion of hospitalization reimbursement $(r=0.229$, $\mathrm{P}<0.01 ; \mathrm{r}=-0.157, \mathrm{P}<0.05$ ). At present, the medical insurance in most provinces and cities in China only reimburses the hospitalization expenses of patients with acute stroke, and the patients and their family members have to pay out-ofpocket for the rehabilitation. Due to economic status and medical resource allocation, most stroke patients choose to go home for rehabilitation after spending the acute phase in the hospital, and their family members offer almost all their home-based care (17). The patient's dependency further increases care stress. Thus, the government authorities should pay special attention to stroke patients and their caregivers by optimizing relevant welfare policies, easing the stress status of caregivers, and improving the quality of care. Also, rehabilitation hospitals, governments, volunteers, and other stakeholders may organize personnel to offer home-based rehabilitation for discharged patients, improve the ability of patients' family members to care for patients at home for economic reasons, and further alleviate the caring stress during the care of disabled patients.

The patient's dependency was negatively correlated with care stress $(\mathrm{r}=0.258, \mathrm{P}<0.01)$. Thus, family members of patients with "mild dependency" are less likely to be at highstress status. For patients with higher ADL dependency, 
the caregivers have to spend more time, energy, physical strength, and financial expenditure during the care and rehabilitation, along with fewer social activities and working hours in their own life and work, which ultimately increases the caring stress.

Treatment decision-making is a complex process with many uncertainties and risks (18). Family members often function as a surrogate decision-maker for stroke patients (18). During the decision-making, family members will experience strong negative emotions, including learned helplessness, anxiety, and regret $(19,20)$, and as surrogate decision-makers, they have to endure huge stress $(21,22)$. However, the results of our current study showed that family members served as a "completely surrogate decisionmaker" in the treatment decision-making had lower selfperceived stress than those who were "not participate in decision-making at all." A possible explanation is: all the above literature reported the stress experienced by the surrogate decision-makers when they were making critical decisions (e.g., maintaining a life-supporting therapy or not) for patients with serious or irreversible progressive diseases. In our current study, however, the surrogate decision-makers participated in decision-making on daily treatment and nursing and thus had lower ethical stress. Another reason may be that, in our current study, most family members $(94.8 \%)$ were the payer of the patient's hospital expenditures. They controlled the hospitalization expenditures by controlling the adoption of treatment and nursing services. When the family members had solid knowledge about the payment modes and details, they were less likely to experience high financial pressure arising from unknown hospital care and its costs.

\section{Optimizing family support for disabled stroke patients}

When caring for a patient, medical staff should not only treat diseases but treat the patient. The role of family support should be fully recognized, and effective measures should be taken to enhance family support. Unfortunately, the family support of patients gradually decreases with the prolonged disease course (23). And the family members' stress will directly affect their attitudes towards the patient and quality of care (4) and decrease family support. Family support includes information support, emotional support, and behavioral support. Family member's participation in treatment decision-making is one of the crucial ways of behavioral support for the patients (24) and can enhance family support. A positive family atmosphere can encourage patients to express their thoughts and feelings and alleviate their loneliness (25). Effective and healthy communication between caregivers and patients is essential for the physical and mental recovery of the patients (25). Meanwhile, strategies including knowledge training, empowerment/ guidance, home-based rehabilitation should be taken to improve home-based support for disabled stroke patients.

\section{Conclusions}

A substantial proportion of family members are under high stress when caring for disabled stroke patients. The high-stress levels of the patient's family members not only reduce their quality of life but also directly affects the patient's emotions, which affects the patient's compliance with rehabilitation and treatment and even causes or aggravates the patient's depression (5). Strategies including health education, empowerment/guidance, home-based rehabilitation, and information and emotional support may effectively relieve the caring stress, improve the family support of patients, and improve the quality of life of both patients and caregivers. Meanwhile, medical insurers, government authorities, and other stakeholders should perfect the relevant welfare policies and offer specific financial support for the rehabilitation of disabled stroke patients. The aim is to lower the financial pressure of family caregivers, improve the family support for disabled stroke patients, alleviate the patient's depressed mood, promote the confidence of the patients on subsequent rehabilitation, increase their rehabilitation compliance, and ultimately improve the rehabilitation effectiveness.

\section{Acknowledgments}

Funding: None.

\section{Footnote}

Reporting Checklist: The authors have completed the MDARreporting checklist. Available at http://dx.doi. org/10.21037/apm-20-1369

Data Sharing Statement: Available at http://dx.doi. org/10.21037/apm-20-1369

Conflicts of Interest: All authors have completed the ICMJE uniform disclosure form (available at http://dx.doi. org/10.21037/apm-20-1369). The authors have no conflicts 
of interest to declare.

Ethical Statement: The authors are accountable for all aspects of the work in ensuring that questions related to the accuracy or integrity of any part of the work are appropriately investigated and resolved. This study was approved by the medical Ethics Committee of Hainan Cadres Sanatorium (Hainan Geriatric Hospital). The study conformed to the provisions of the Declaration of Helsinki (as revised in 2013). Informed consent was taken from all the patients.

Open Access Statement: This is an Open Access article distributed in accordance with the Creative Commons Attribution-NonCommercial-NoDerivs 4.0 International License (CC BY-NC-ND 4.0), which permits the noncommercial replication and distribution of the article with the strict proviso that no changes or edits are made and the original work is properly cited (including links to both the formal publication through the relevant DOI and the license). See: https://creativecommons.org/licenses/by-nc-nd/4.0/.

\section{References}

1. Sarfo FS, Ovbiagele, B. Stroke minimization through additive anti-atherosclerotic agents in routine treatment (SMAART): a pilot trial concept for improving stroke outcomes in sub-saharan Africa. J Neurol Sci 2017;377:167-73.

2. Gu HQ, Yang X, Rao ZZ, et al. Disparities in outcomes associated with rural-urban insurance status in China among inpatient women with stroke: a registry-based cohort study. Ann Transl Med 2019;7:426.

3. Jamison J, Jonathan G, Ricky M, et al. Stroke survivors', caregivers' and GPs' attitudes towards a polypill for the secondary prevention of stroke: a qualitative interview study. BMJ Open 2016;6:e010458.

4. White CL, Brady TL, Saucedo LL, et al. Towards a better understanding of readmissions after stroke: partnering with stroke survivors and caregivers. J Clin Nurs 2015;24:1091100.

5. Aziz NA, Pindus DM, Mullis R, et al. Understanding stroke survivors' and informal carers' experiences of and need for primary care and community health services-a systematic review of the qualitative literature: protocol. BMJ Open 2016;6:e009244

6. Mahoney FI, Barthel DW. Functional evaluation: the barthel index. Md State Med J 1965;14:61-5.
7. Oveisgharan S, Shirani S, Ghorbani A, et al. Barthel index in a Middle-East country: translation, validity and reliability. Cerebrovasc Dis 2006;22:350-4.

8. Cameron JI, Naglie G, Silver FL, et al. Stroke family caregivers' support needs change across the care continuum: a qualitative study using the timing it right framework. Disabil Rehabil 2013;35:315-24.

9. Yang $\mathrm{XY}, \mathrm{Xu} \mathrm{W}, \mathrm{Xu} \mathrm{YH}$. A questionnaire survey on the mental health status of caregivers of hospitalized rehabilitation stroke patients. Chin J Rehabil Med 2012;27:1145-6.

10. Wang ZL, Zhao Y. Correlation analysis of comprehensive care ability and care pressure of stroke carers. Nurs Res 2012;26:690-1.

11. Donaghy E, Salisbury L, Lone NI, et al. Unplanned early hospital readmission among critical care survivors: a mixed methods study of patients and carers. BMJ Qual Saf 2018;27:915-27.

12. Mackenzie A, Greenwood N. Positive experiences of caregiving in stroke: a systematic review. Disabil Rehabil 2012;34:1413-22.

13. Willeke WJ, Visser-Meily JMA, Post MWM, et al. Positive caregiving experiences are associated with life satisfaction in spouses of stroke survivors. J Stroke Cerebrovasc Dis 2012;21:801-7.

14. Krieger T, Feron F, Dorant E, et al. Developing a complex intervention programme for informal caregivers of stroke survivors: The Caregivers' Guide. Scand J Caring Sci 2017;31:146-56.

15. Czerwonka AI, Herridge MS, Chan L, et al. Changing support needs of survivors of complex critical illness and their family caregivers across the care continuum: a qualitative pilot study of Towards RECOVER. J Crit Care 2015;30:242-9.

16. Gholamzadeh S, TengkuAizan H, Sharif F, et al. Exploration the supportive needs and coping behaviors of daughter and daughter-in-law caregivers of stroke survivors, Shiraz-Iran: a qualitative content analysis. Int J Community-based Nurs Midwifery 2015;3:205-15.

17. Chau JPC, Lo SHS, Lee VWY, et al. Effectiveness and cost-effectiveness of a virtual multidisciplinary stroke care clinic for community-dwelling stroke survivors and caregivers: a randomised controlled trial protocol. BMJ Open 2019;9:e026500.

18. Akosile CO, Banjo TO, Okoye EC, et al. Informal caregiving burden and perceived social support in an acute stroke care facility. Health Qual Life Outcomes 2018;16:57. 
19. Kuniavsky M, vanHeerden PV, Kadmon I, et al. Attitudes of legal guardians in the ICU: a qualitative report. Intensive Crit Care Nurs 2014;30:86-92.

20. Hines SC, Glover JJ, Babrow AS, et al. Improving advance care planning by accommodating family preferences. J Palliat Med 2001;4:481-9.

21. Qiu X, Sit JWH, Koo FK, et al. The influence of Chinese culture on family caregivers of stroke survivors: A qualitative study. J Clin Nurs 2018;27:e309-19.

22. Pindus DM, Mullis R, Lim L, et al. Stroke survivors' and informal caregivers' experiences of primary care and community healthcare services-A systematic review and

Cite this article as: $\mathrm{Wu} \mathrm{X}$, Liang $\mathrm{Y}$, Zheng $\mathrm{B}$, Wang $\mathrm{H}$, Ning N, Zheng H, Shi B. Care stress in caregivers of disabled stroke patients: a cross-sectional survey. Ann Palliat Med 2020;9(4):2211-2220. doi: 10.21037/apm-20-1369 meta-ethnography. PLoS One 2018;13:e0192533.

23. Qiu Y, Li S, Qiu Y, et al. Stroke: coping strategies and depression among Chinese caregivers of survivors during hospitalisation. J Clin Nurs 2008;17:1563-73.

24. Camak DJ. Addressing the burden of stroke caregivers: a literature review. J Clin Nurs 2015;24:2376-82.

25. Ardahan M, Yesilbalkan OU. Perceived family support of women with breast cancer and affecting factors in turkey. Asian Pac J Cancer Prev 2010;11:1425-9.

(English Language Editor: J. Chapnick) 